\title{
Leveraging latest computer science tools to advance nuclear cardiology
}

\author{
Piotr Slomka, PhD \\ a Department of Medicine, Cedars-Sinai Medical Center, Los Angeles, CA
}

Received Aug 18, 2019; accepted Aug 19, 2019

doi: $10.1007 / \mathrm{s} 12350-019-01873-y$

\begin{abstract}
Nuclear cardiology has unique advantages compared to other modalities, since the image analysis is already much more automated compared to what is currently clinically performed for CT, MR, or echocardiography imaging. The diverse image and clinical data available to assess coronary disease function, perfusion, flow, and associated $\mathrm{CT}$ data provide new opportunities, but logistically these additional assessments increase the overall complexity of SPECT/ PET reporting, necessitating additional expertise and time. The advances in artificial intelligence software can be leveraged to obtain comprehensive risk predictions and diagnoses from all available data. They will allow nuclear cardiology to retain competitive edge compared to other modalities and improve its overall clinical utility. These tools will enhance diagnosis and risk prediction beyond what is possible by subjective visual analysis and mental integration of data by physicians.
\end{abstract}

Noninvasive imaging is widely used for the diagnosis, risk assessment, and management of coronary artery disease (CAD) - providing crucial information about myocardial perfusion, function, and anatomy. Myocardial perfusion imaging (MPI) by SPECT and PET is widely used, with 15 to 20 million SPECT MPI scans being performed annually worldwide. ${ }^{1-3}$ In addition, noncontrast $\mathrm{CT}$ can reliably detect coronary artery calcium (CAC), a specific marker of coronary atherosclerosis, providing complementary information to MPI. SPECT and PET scanners have evolved significantly in recent years, ${ }^{4,5}$ allowing for simultaneous gains in sensitivity and resolution. ${ }^{6,7}$ Many new systems come hybrid configuration with CT scanners for CTbased attenuation correction (CTAC). In parallel, image reconstruction methods with resolution recovery and time-of-flight for PET have enhanced image quality. While CTAC maps are obtained without ECG-gating, it is possible to extract useful CAC information from

Reprint requests: Piotr Slomka, PhD, Department of Medicine, CedarsSinai Medical Center, 8700 Beverly Boulevard, Ste. A047N, Los Angeles, CA 90048; slomkap@cshs.org

J Nucl Cardiol 2019;26:1501-4.

$1071-3581 / \$ 34.00$

Copyright (C) 2019 American Society of Nuclear Cardiology. them. ${ }^{8,9}$ It has been shown that scores obtained automatically from PET CTAC correlate well with CAC scores from standard, ECG-gated CAC scans ${ }^{10}$ and that visual identification of CAC on CTAC scans improves the diagnostic accuracy of MPI. ${ }^{11}$

While regional relative quantification of total perfusion deficit (TPD) and ischemia detection by MPI are in wide clinical use, these approaches may underestimate extensive $\mathrm{CAD}$ - most notably when there is global diminution of myocardial blood flow (MBF). MBF by PET complements perfusion imaging, with diagnostic ${ }^{12}$ and prognostic information. ${ }^{13,14}$ Additionally, PET MBF improves diagnosis of CAD over CAC and relative perfusion. ${ }^{15}$ However, currently SPECT is used more widely than PET-representing over $95 \%$ of MPI. ${ }^{16}$ Can MBF also be estimated from SPECT? Many recent reports have confirmed the feasibility of SPECT flow measurement with an early scan. ${ }^{17-24}$ Several sites are commencing clinical SPECT flow protocols. PET flow tools are being adapted and validated for SPECT flow measurements. Intriguingly, aside from flow, the early SPECT scan allows measurement of functional reserve at peak stress ${ }^{25}$-a robust marker of multivessel disease in PET. ${ }^{26}$ The flow measurements need to be considered together with standard perfusion and 
function measurements to provide the final diagnostic and prognostic assessment.

MPI is extensively validated for risk assessment, but the main supporting studies utilized visual analysis. $^{27,28}$ However, recently the quantitative risk stratification by quantitively measures was performed. ${ }^{29-31}$ In a large multicenter study, we showed that TPD allows more precise risk gradation for MACE, even in categories traditionally considered normal. ${ }^{32}$ However, most MPI risk-stratification studies used perfusion defect size alone, without considering other imaging (PET flow, CAC), stress test, or clinical variables-or considered only subset of these variables. While physicians mentally attempt to combine all stress test and clinical variables when interpreting MPI, such integration is subjective and affected by individual bias and expertise. There are currently no integrated guidelines on how to use all this new imaging information holistically to provide diagnostic and treatment recommendation after the MPI test.

In recent years, new highly efficient artificial intelligence (AI) tools have emerged. These methods are radically reshaping many fields, including health care. New AI-based approaches are revolutionizing image analysis and could further enhance MPI interpretation. In particular, convolutional neural networks $(\mathrm{CNN})-\mathrm{a}$ subclass of "deep learning" - gained considerable attention $^{33}$ due to significantly improved classification of natural images. ${ }^{34}$ These new tools can be efficiently leveraged for direct extraction of information from MPI and CT, eliminating human subjectivity. Few studies now showed that deep learning can detect CAD directly from polar maps, ${ }^{35-37}$ outperforming current quantitative methods, both on a regional and per-patient basis, ${ }^{35}$ including joint analysis of multiple maps, ${ }^{36}$ which is a visually challenging task.

The diverse image and clinical data available to assess CAD function from MPI, perfusion, flow, and associated CT data provide new opportunities, but logistically these additional assessments increase the overall complexity of SPECT/PET reporting, necessitating additional expertise and time. Consequently, the burden placed on nuclear cardiology physicians is increasing. They are required to constantly improve the objectivity and precision of their findings with this additional information-while absorbing the cost. It seems that the natural approach to consider all this available information together to provide a final precise diagnosis and risk estimate tailored to the specific patient is to leverage the latest AI methods developed in computer vision and machine learning fields.

Nuclear cardiology has unique advantages compared to other modalities, since the image analysis is already much more automated compared to what is currently clinically performed for CT, MRI or echocardiography imaging. Large imaging registries with MPI images and outcomes are available for fully automated analysis. ${ }^{38}$ The images are small in size and well standardized allowing implementation of clinically practical software. While there is an ongoing research in automation of CT angiography, MRI, and echocardiography, the current clinical levels of automation and quantitation in nuclear cardiology is probably at least 5 to 10 years ahead, compared to other cardiovascular modalities. Standard image processing of nuclear cardiology images demonstrated already diagnostic equivalence to experienced readers in large studies. ${ }^{39}$ Recently, new AI tools have been proposed for nuclear cardiology. Machine learning has been demonstrated to achieve improved prediction of disease and outcomes from SPECT MPI, by combining imaging ${ }^{40}$ and clinical parameters. ${ }^{41-43}$ AI has been deployed to create final clinical reports. ${ }^{44}$ In studies using clinical and SPECT MPI data to predict revascularization, AI had similar to, or better performance than, expert visual interpretation. ${ }^{43,45}$ AI surpassed clinical interpretation of MPI for MACE prediction. ${ }^{42}$ These studies demonstrate the significant gains in disease diagnosis and prognostication attainable by integrating imaging and clinical data.

Where is it going? The advances in software allowing comprehensive risk prediction and diagnosis from all available data (e.g., clinical data flow perfusion, function, CTAC) will allow nuclear cardiology to retain competitive edge compared to other modalities and improve its overall clinical utility. These tools will improve risk prediction beyond what is possible by subjective visual analysis and mental integration of data by physicians. The increased precision and accuracy of novel software strategies will provide a paradigmchanging solution for nuclear cardiology. These developments will not threaten nuclear cardiologists' jobs. Rather, these developments will provide a new tool in the physicians' arsenal to improve the accuracy of their diagnosis, and enable them to select the appropriate therapy for their patients in a scientific, precise, and quantitative manner. The precise quantitative results can be presented to clinicians (and patients) in easy-to-understand terms (e.g., \% risk per year), or as the relative risk of one therapy compared to the alternative for a specific patient. The same quality and quantitative precision will be available regardless which part of the world the studies were performed. Surely, other imaging modalities will follow the same path, but nuclear cardiology is in the position to be the vanguard of these upcoming changes in medical imaging. 


\section{Disclosures}

The author participates in software royalties for nuclear cardiology software at the Cedars-Sinai Medical Center and have received research grant support from the Siemens Medical Systems.

\section{References}

1. IMV. Nuclear medicine market outlook report 2018. IMV, 2019. h ttps://imvinfo.com/product/nuclear-medicine-market-outlook-repo rt-2018/.

2. Einstein AJ, Pascual TN, Mercuri M, et al. Current worldwide nuclear cardiology practices and radiation exposure: Results from the 65 country IAEA Nuclear Cardiology Protocols Cross-Sectional Study (INCAPS). Eur Heart J 2015;36:1689-96.

3. Mercuri M, Pascual TN, Mahmarian JJ, et al. Estimating the reduction in the radiation burden from nuclear cardiology through use of stress-only imaging in the United States and worldwide. JAMA Intern Med 2016;176:269-73.

4. Slomka P, Miller RJ, Hu LH, Germano G, Berman D. Solid-state detector SPECT myocardial perfusion imaging. J Nucl Med 2019;1:1. https://doi.org/10.2967/jnumed.118.220657.

5. Slomka PJ, Pan T, Germano G. Recent advances and future progress in PET instrumentation. Semin Nucl Med 2016;46:5-19.

6. Sharir T, Ben-Haim S, Merzon K, et al. High-speed myocardial perfusion imaging: Initial clinical comparison with conventional dual detector anger camera imaging. JACC Cardiovasc Imaging 2008;1:156-63.

7. Imbert L, Poussier S, Franken PR, et al. Compared performance of high-sensitivity cameras dedicated to myocardial perfusion SPECT: A comprehensive analysis of phantom and human images. J Nucl Med 2012;53:1897-903.

8. Einstein AJ, Johnson LL, Bokhari S, et al. Agreement of visual estimation of coronary artery calcium from low-dose CT attenuation correction scans in hybrid PET/CT and SPECT/CT with standard Agatston score. J Am Coll Cardiol 2010;56:1914-21.

9. Kaster TS, Dwivedi G, Susser L, et al. Single low-dose CT scan optimized for rest-stress PET attenuation correction and quantification of coronary artery calcium. J Nucl Cardiol 2015;22:419-28.

10. Isgum I, de Vos BD, Wolterink JM, et al. Automatic determination of cardiovascular risk by $\mathrm{CT}$ attenuation correction maps in $\mathrm{Rb}-82$ PET/CT. J Nucl Cardiol 2017;25:2133-42.

11. Patchett ND, Pawar S, Miller EJ. Visual identification of coronary calcifications on attenuation correction CT improves diagnostic accuracy of SPECT/CT myocardial perfusion imaging. J Nucl Cardiol 2017;24:711-20.

12. Naya M, Murthy VL, Taqueti VR, et al. Preserved coronary flow reserve effectively excludes high-risk coronary artery disease on angiography. J Nucl Med 2014;55:248-55.

13. Murthy VL, Naya M, Foster CR, et al. Improved cardiac risk assessment with noninvasive measures of coronary flow reserve. Circulation 2011;124:2215-24.

14. Ziadi MC, Dekemp RA, Williams KA, et al. Impaired myocardial flow reserve on rubidium-82 positron emission tomography imaging predicts adverse outcomes in patients assessed for myocardial ischemia. J Am Coll Cardiol 2011;58:740-8.

15. Zampella E, Acampa W, Assante R, et al. Combined evaluation of regional coronary artery calcium and myocardial perfusion by (82) Rb PET/CT in the identification of obstructive coronary artery disease. Eur J Nucl Med Mol Imaging 2018;45:521-9.
16. Levin DC, Parker L, Halpern EJ, Rao VM. Recent trends in imaging for suspected coronary artery disease: What is the best approach? JACR 2016;13:381-6.

17. Ben-Haim S, Murthy VL, Breault C, et al. Quantification of myocardial perfusion reserve using dynamic SPECT imaging in humans: A feasibility study. J Nucl Med 2013;54:873-9.

18. Wells RG, Marvin B, Poirier M, Renaud J, de Kemp RA, Ruddy TD. Optimization of SPECT measurement of myocardial blood flow with corrections for attenuation, motion, and blood binding compared with PET. J Nucl Med 2017;58:2013-9.

19. Wells RG, Timmins R, Klein R, et al. Dynamic SPECT measurement of absolute myocardial blood flow in a porcine model. $\mathrm{J}$ Nucl Med 2014;55:1685-91.

20. Agostini D, Roule V, Nganoa C, et al. First validation of myocardial flow reserve assessed by dynamic $(99 \mathrm{~m}) \mathrm{Tc}$-sestamibi CZT-SPECT camera: Head to head comparison with (15)O-water PET and fractional flow reserve in patients with suspected coronary artery disease. The WATERDAY study. Eur J Nucl Med Mol Imaging 2018;45:1079-90.

21. Ma R, Wang L, Wu D, et al. Myocardial blood flow quantitation in patients with congestive heart failure: Head-to-head comparison between rapid-rotating gantry SPECT and CZT SPECT. J Nucl Cardiol 2019. https://doi.org/10.1007/s12350-019-01621-2.

22. Nkoulou R, Fuchs TA, Pazhenkottil AP, et al. Absolute myocardial blood flow and flow reserve assessed by gated SPECT with Cadmium-Zinc-Telluride detectors using 99mTc-tetrofosmin: Head-to-head comparison with $13 \mathrm{~N}$-ammonia PET. J Nucl Med 2016;57:1887-92.

23. Ben Bouallegue F, Roubille F, Lattuca B, et al. SPECT myocardial perfusion reserve in patients with multivessel coronary disease: Correlation with angiographic findings and invasive fractional flow reserve measurements. J Nucl Med 2015;56:1712-7.

24. Otaki Y, Manabe O, Miller RD et al. Quantification of myocardial blood flow by CZT SPECT with motion correction and comparison with 15O-water PET. J Nucl Cardiol 2019; (in press).

25. Brodov Y, Fish M, Rubeaux M, et al. Quantitation of left ventricular ejection fraction reserve from early gated regadenoson stress Tc-99m high-efficiency SPECT. J Nucl Cardiol 2016;23:1251-61

26. Dorbala S, Vangala D, Sampson U, Limaye A, Kwong R, Di Carli MF. Value of vasodilator left ventricular ejection fraction reserve in evaluating the magnitude of myocardium at risk and the extent of angiographic coronary artery disease: A $82 \mathrm{Rb}$ PET/CT study. J Nucl Med 2007;48:349-58.

27. Berman DS, Hachamovitch R, Kiat $\mathrm{H}$, et al. Incremental value of prognostic testing in patients with known or suspected ischemic heart disease: A basis for optimal utilization of exercise technetium-99m sestamibi myocardial perfusion single-photon emission computed tomography. J Am Coll Cardiol 1995;26:63947.

28. Hachamovitch R, Berman DS, Shaw LJ, et al. Incremental prognostic value of myocardial perfusion single photon emission computed tomography for the prediction of cardiac death: Differential stratification for risk of cardiac death and myocardial infarction. Circulation 1998;97:535-43.

29. Motwani M, Leslie WD, Goertzen AL, et al. Fully automated analysis of attenuation-corrected SPECT for the long-term prediction of acute myocardial infarction. $\mathrm{J}$ Nucl Cardiol 2018;25:1353-60.

30. Xu Y, Nakazato R, Hayes S, et al. Prognostic value of automated vs visual analysis for adenosine stress myocardial perfusion SPECT in patients without prior coronary artery disease: A casecontrol study. J Nucl Cardiol 2011;18:1003-9 Epub 2011 Sep 20. 
31. Otaki Y, Betancur J, Sharir T et al. 5-Year prognostic value of quantitative vs visual myocardial perfusion imaging in subtle perfusion defects: Results from the REFINE SPECT registry. JACC Cardiovasc Imaging 2019; (in press).

32. Otaki Y, Betancur J, Sharir T, et al. 5-Year prognostic value of quantitative versus visual MPI in subtle perfusion defects: Results from REFINE SPECT. JACC Cardiovasc Imaging 2019. https://d oi.org/10.1016/j.jcmg.2019.02.028.

33. Sharif Razavian A, Azizpour H, Sullivan J, Carlsson S. CNN features off-the-shelf: An astounding baseline for recognition. In: Proceedings of the IEEE conference on computer vision and pattern recognition workshops. 2014;806-813.

34. Krizhevsky A, Sutskever I, Hinton GE. Imagenet classification with deep convolutional neural networks. Adv Neural Inform Process Syst 2012;1097-105.

35. Betancur J, Commandeur F, Motlagh M, et al. Deep learning for prediction of obstructive disease from fast myocardial perfusion SPECT: A multicenter study. JACC Cardiovasc Imaging 2018;11:1654-63.

36. Betancur JA, Hu LH, Commandeur F, et al. Deep learning analysis of upright-supine high-efficiency SPECT myocardial perfusion imaging for prediction of obstructive coronary artery disease: A multicenter study. J Nucl Med 2018. https://doi.org/10.2967/jnu med.118.213538.

37. Spier N, Nekolla S, Rupprecht C, Mustafa M, Navab N, Baust M. Classification of polar maps from cardiac perfusion imaging with graph-convolutional neural networks. Sci Rep 2019;9:7569.

38. Slomka PJ, Betancur J, Liang JX, et al. Rationale and design of the REgistry of Fast Myocardial Perfusion Imaging with NExt generation SPECT (REFINE SPECT). J Nucl Cardiol 2018. https://d oi.org/10.1007/s12350-018-1326-4.
39. Arsanjani R, Xu Y, Hayes SW, et al. Comparison of fully automated computer analysis and visual scoring for detection of coronary artery disease from myocardial perfusion SPECT in a large population. J Nucl Med 2013;54:221-8.

40. Arsanjani R, Xu Y, Dey D, et al. Improved accuracy of myocardial perfusion SPECT for the detection of coronary artery disease using a support vector machine algorithm. J Nucl Med 2013;54:549-55.

41. Arsanjani R, Xu Y, Dey D, et al. Improved accuracy of myocardial perfusion SPECT for detection of coronary artery disease by machine learning in a large population. J Nucl Cardiol 2013;20:553-62.

42. Betancur J, Otaki Y, Motwani M, et al. Prognostic value of combined clinical and myocardial perfusion imaging data using machine learning. JACC Cardiovasc Imaging 2018;11:1000-9.

43. Hu LH, Betancur J, Sharir T, et al. Machine learning predicts pervessel early coronary revascularization after fast myocardial perfusion SPECT: Results from multicentre REFINE SPECT registry. European heart journal cardiovascular Imaging 2019. https://doi. org/10.1093/ehjci/jez177/5533091.

44. Garcia EV, Klein JL, Moncayo V, et al. Diagnostic performance of an artificial intelligence-driven cardiac-structured reporting system for myocardial perfusion SPECT imaging. J Nucl Cardiol 2018. h ttps://doi.org/10.1007/s12350-018-1432-3.

45. Arsanjani R, Dey D, Khachatryan T, et al. Prediction of revascularization after myocardial perfusion SPECT by machine learning in a large population. J Nucl Cardiol 2015;22:877-84.

Publisher's Note Springer Nature remains neutral with regard to jurisdictional claims in published maps and institutional affiliations. 